\title{
Diuretic of Choice in ABFM Hypertension Self-Assessment Module Simulations
}

\author{
Michael D. Hagen, MD, Walton Sumner, MD, and Haiqi Fu, MS
}

Background: Evidence suggests that chlorthalidone has therapeutic advantages over hydrochlorothiazide, perhaps because of a longer antihypertensive effect. Although guidelines such as the Seventh Report of the Joint National Committee on Prevention, Detection, Evaluation, and Treatment of High Blood Pressure treat diuretics as a class, some experts believe chlorthalidone is the diuretic of choice in hypertension management. We evaluated diplomates' use of chlorthalidone and hydrochlorothiazide as first-choice diuretics in virtual patient simulations of hypertension in the American Board of Family Medicine Maintenance of Certification for Family Physicians self-assessment modules.

Methods: We examined action logs for hypertension simulations completed between 2004 and 2011 and identified initial antihypertensive choices made by diplomates. We tabulated uses of any diuretic as initial treatment, distinguishing between chlorthalidone, hydrochlorothiazide, and other diuretic choices. We examined trends in the use of diuretics and chlorthalidone in simulations using linear models.

Results: Chlorthalidone use increased 0.7 percentage points per year (test for $\beta>0, P<.0013$ ), from approximately $1.3 \%$ of simulation prescriptions in 2004 to about $4.8 \%$ in 2010 and 2011 , whereas hydrochlorothiazide prescriptions fell 2 percentage points per year $(P=.035)$, from nearly $57 \%$ to 47\%. As a fraction of all diuretic use, chlorthalidone increased 1.4 percentage points per year $(P=.0006)$, from $2 \%$ to $9 \%$.

Conclusions: Small but growing numbers of diplomates are heeding recommendations in the growing literature to start with chlorthalidone rather than hydrochlorothiazide, at least in the virtual patient environment. Observed choices in virtual patient management strongly imply that this is a valid topic for additional attention in the hypertension self-assessment module. (J Am Board Fam Med 2012;25:

805-809.)

Keywords: Chlorthalidone, Hydrochlorothiazide, Hypertension, Thiazide Diuretics

The Seventh Report of the Joint National Committee on Prevention, Detection, Evaluation, and Treatment of High Blood Pressure (JNC 7) emphasized the role of thiazide diuretics as initial therapy for

This article was externally peer reviewed.

Submitted 28 December 2011; revised 20 June 2012; accepted 25 June 2012.

From the American Board of Family Medicine, Lexington, KY (MDH, HF); the Department of Family and Community Medicine, University of Kentucky College of Medicine, Lexington, KY (MDH); and Department of Internal Medicine, Washington University School of Medicine, St. Louis, MO (WS).

Funding: This work was funded by the American Board of Family Medicine.

Conflict of interest: none declared.

Corresponding author: Michael D. Hagen, MD, American Board of Family Medicine, 1648 McGrathiana Parkway, Suite 550, Lexington, KY 40511 (E-mail: hagen@ theabfm.org). hypertension. ${ }^{1}$ In the United States, the thiazide diuretic hydrochlorothiazide remains the most frequently prescribed antihypertensive agent. ${ }^{2}$ However, a number of authors recently have called for reexamination of this choice, pointing out that the original studies demonstrating diuretic antihypertensive efficacy used chlorthalidone. ${ }^{2-7}$

Since 2004 the American Board of Family Medicine has offered a hypertension self-assessment module (SAM) in the Maintenance of Certification for Family Physicians program. ${ }^{8}$ The SAM consists of 2 parts: a 60-item knowledge assessment and a clinical simulation that focuses on management of a patient with hypertension. Both the knowledge assessment and clinical simulation have emphasized the recommendations presented in the JNC 7 and have not included explicit information reflecting 
the recent literature suggestions regarding thiazide diuretic choice. Actions taken during the clinical simulation provide a potential probe regarding the diffusion into clinical practice of new information from sources other than Maintenance of Certification for Family Physicians materials. Unlike most patients encountered in practice, these virtual patients do not present with established treatment regimens, and physicians can use their preferred agent as initial therapy. We explored the prescribing behaviors used in hypertension simulations completed since 2004 to see if American Board of Family Medicine Diplomates incorporated these considerations in the management of hypertension, at least in a virtual patient environment.

\section{Methods}

We identified hypertension simulations completed between 2004 and 2011. The record of each completed simulation includes a $\log$ of all actions taken. During a simulation, drug prescriptions always have been selected by typing a few characters from the drug name and pressing the Enter key or clicking a search button. The system returns a list of drug names containing the search string. For instance, "thi" and "thiazide" match "hydrochlorothiazide" but not "chlorthalidone." "HCTZ" uniquely matches the corresponding brand name. Thus, chlorthalidone selections require a deliberate search for that agent. Each action (identified in the system as a "Course of Action," or COA) has an associated COA identification code (COA ID, eg, "Hydrochlorothiazide 14699"). We searched the action logs for COA IDs of all diuretics (single and combination products). We counted only the first antihypertensive prescription in each simulation. We report the number and percentage of simulations in which diuretics were used and which diuretic was used. Linear least squares models were fitted using JMP statistical software (version 5.01a, SAS Institute Inc, Cary, NC) to predict hydrochlorothiazide use, chlorthalidone use, and the fraction of diuretic prescriptions that used chlorthalidone as a function of year, from 2004 to 2011, weighted by the number of simulations. The $\beta$ coefficients of the model for the year variable were tested for being significantly different from zero.

\section{Results}

Diplomates completed 37,843 distinct hypertension simulations between 2004 and 2011. Table 1 enumerates diuretic prescriptions written each year during hypertension simulations.

Chlorthalidone use rose 0.7 percentage points per year (test for $\beta$ coefficient $>0, P<.0013$ ), from $1.3 \%$ in 2004 ( $2 \%$ of all diuretic use) to $4.8 \%$ by 2010 (9\% of all diuretic use; Figure 1). Conversely, hydrochlorothiazide initially rose from $57 \%$ in 2004 to a high of $60 \%$ in 2005 (nearly $100 \%$ of diuretic use) but declined, on average, 2 percentage points per year $(P=.035)$ to approximately $48 \%$ by 2010 (70\% of diuretic use). As a fraction of all diuretic use, chlorthalidone increased 1.4 percentage points per year $(P=.0006)$, from $2 \%$ to $9 \%$. Residual plots were randomly distributed around zero.

\section{Discussion}

The hypertension simulation experience suggests that, at least in the virtual patient environment,

Table 1. Diuretics as Initial Antihypertensive Prescriptions during the Management of Virtual Patients Portraying Hypertension.

\begin{tabular}{|c|c|c|c|c|c|c|}
\hline Year & Simulations & HCTZ & HCTZ and Second Diuretic & HCTZ and Other Agent & Chlorthalidone & Other Diuretics \\
\hline 2004 & 1863 & 935 & 72 & 62 & 24 & 2 \\
\hline 2005 & 5124 & 2771 & 187 & 98 & 45 & 3 \\
\hline 2006 & 3730 & 1822 & 131 & 72 & 45 & 3 \\
\hline 2007 & 3545 & 1684 & 116 & 71 & 57 & 3 \\
\hline 2008 & 4595 & 2020 & 132 & 138 & 75 & 3 \\
\hline 2009 & 5511 & 1813 & 110 & 112 & 151 & 3 \\
\hline 2010 & 6762 & 2776 & 182 & 163 & 327 & 9 \\
\hline 2011 & 6713 & 2889 & 118 & 149 & 320 & 3 \\
\hline
\end{tabular}

HCTZ, hydrochlorothiazide; HCTZ and second diuretic, combination of hydrochlorothiazide and a potassium sparing diuretic; HCTZ and other agent, combination of hydrochlorothiazide and a nondiuretic antihypertensive agent; other diuretics, chlorothiazide, furosemide, hydroflumethiazide, indapamide, or spironolactone. 
Figure 1. Diuretic prescriptions in hypertension simulations. The fraction of ABFM simulated hypertension patients receiving diuretic prescriptions from Diplomates in Maintenance of Certification is shown for each year since 2004. Most Diplomates prescribe a diuretic; most commonly used diuretic is hydrochlorothiazide, alone or in combination. Chlorthalidone, is being prescribed with gradually increasing frequency.

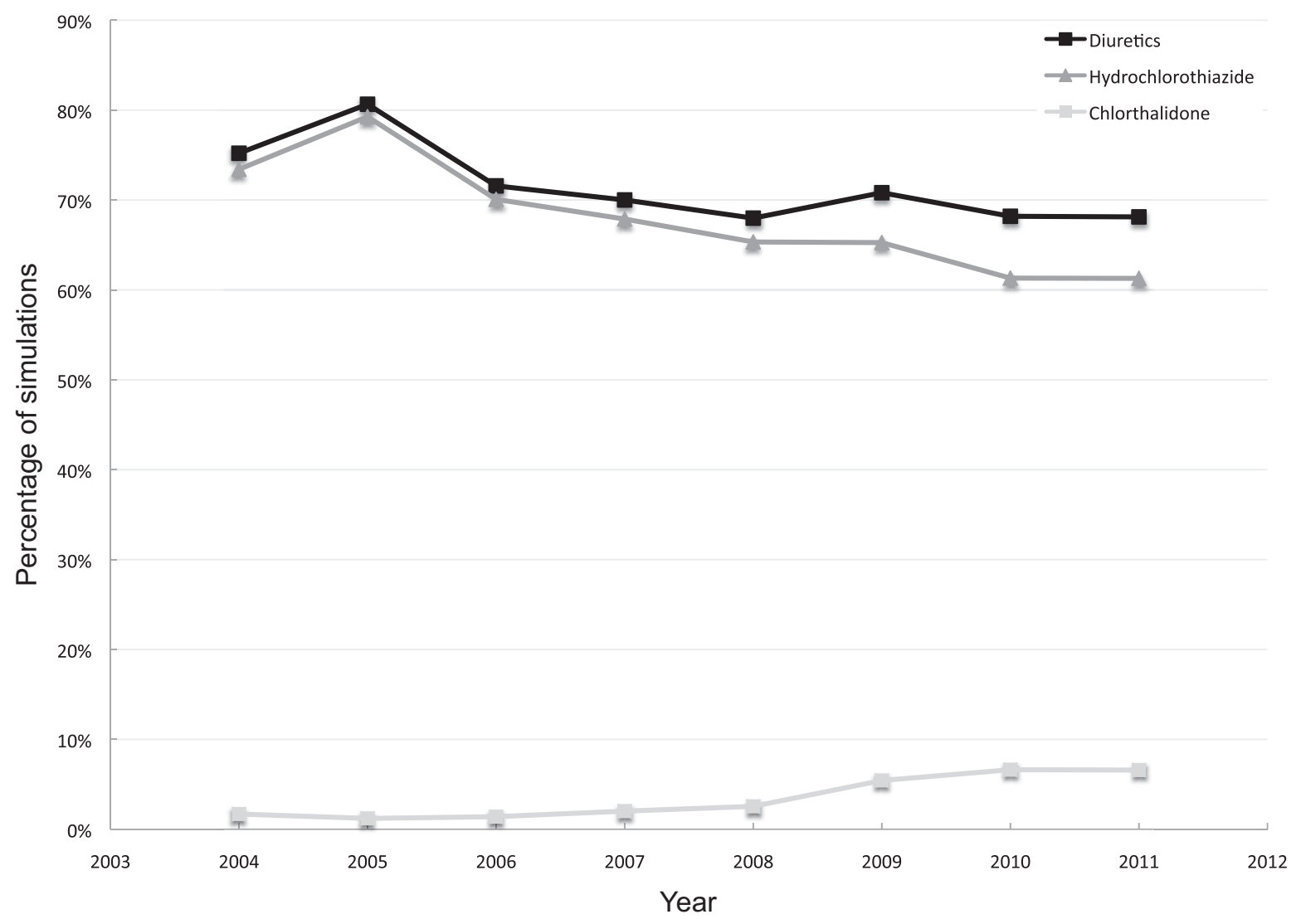

Diplomates have begun to react to the recently published debate regarding thiazide use in hypertensive patients. This has occurred despite the continued heavy emphasis on JNC 7 treatment recommendations in the hypertension SAM.

New findings historically have taken a decade or more to enter clinical practice. ${ }^{9}$ Evidence favoring chlorthalidone over hydrochlorothiazide dates back to 1980 , when the Multiple Risk factor Intervention Trial (MRFIT) stopped administering hydrochlorothiazide in favor of chlorthalidone because of the expected mortality benefits of the latter. ${ }^{10}$ The Systolic Hypertension in the Elderly Program (SHEP), ${ }^{11}$ the Treatment of Moderate Hypertension Study, ${ }^{12}$ and the Antihypertensive and LipidLowering Treatment to Prevent Heart Attack Trial ${ }^{13}$ confirmed the safety and benefits of chlorthalidone. In 2006, Ernst et al ${ }^{14}$ reported that although hydrochlorothiazide provided comparable office measures of blood pressure control, chlorthalidone provided far better overnight control and longer-lasting control. An unexpected observation was that chlorthalidone might be most effective when given before hydrochlorothiazide is used, reinforcing the idea that chlorthalidone should be the first thiazide-like antihypertensive agent for eligible hypertensive patients. Most recently, a 22-year follow-up report of the SHEP participants, whose average age at enrollment was 72 years, found that the intervention recipients gained, on average, 105 days of life expectancy from 4.5 years of chlorthalidone treatment. ${ }^{15}$

The pharmacokinetics of chlorthalidone and hydrochlorothiazide suggest a plausible mechanism for superior results with chlorthalidone. ${ }^{16}$ Both agents start to affect blood pressure approximately 2 hours after initial administration, and reach peak effect within 6 hours. The duration of action of chlorthalidone continues up to 48 hours after a single dose compared with 12 hours for hydrochlorothiazide. Most importantly, the half-life of chlorthalidone 
during long-term administration is between 45 and 60 hours compared with 8 to 15 hours for hydrochlorothiazide. Thus, chlorthalidone lowers blood pressure for days after the last dose; this characteristic could mitigate the harmful consequences of adherence problems and explain chlorthalidone's superior mortality benefit.

Several historical perspectives may account for the persistent preference for hydrochlorothiazide in the United States. Early studies were interpreted to indicate a class effect, and hydrochlorothiazide use was considered a justifiable clinical application of favorable chlorthalidone results, using the quickly written abbreviation and clever brand name "HCTZ." In addition, hydrochlorothiazide has served as the diuretic component of multiple antihypertensive combination drugs, whereas chlorthalidone has been paired with only a few agents, such as atenolol, a relatively ineffective antihypertensive agent. ${ }^{17}$ The implicit endorsement of hydrochlorothiazide and the simple stepped therapy strategies included in the various iterations of the JNC reports almost certainly encourage its use: a patient can start with hydrochlorothiazide and add a potassium-sparing agent, angiotensin-converting enzyme inhibitor, or $\beta$-blocker as needed while filling a single prescription for a combination agent. The lack of a combination of chlorthalidone with potassium-sparing agents seems especially unfortunate given that hypokalemia is the primary complication of chlorthalidone treatment that requires monitoring and limits benefit. ${ }^{18}$ Nevertheless, with excellent generic drugs now available in the most commonly used antihypertensive classes, clinicians can start chlorthalidone and inexpensively add rationally selected agents to create combinations. Observed patterns consistent with this strategy are starting to appear in virtual patient simulations.

We have reported previously that the content of the multiple-choice question section of the asthma SAM seems to influence subsequent decisions during the asthma simulation. ${ }^{19}$ Using multiple-choice questions to introduce concepts followed by rehearsal with virtual patients is a plausible strategy for disseminating compelling ideas more rapidly than through traditional continuing education venues. One of the challenges in this strategy is to identify worthwhile topics. The observation that more than $90 \%$ of Diplomates' initial diuretic prescriptions in virtual patient simulations are for a drug that for more than 30 years has been consid- ered by experts to be inferior to chlorthalidone suggests that this could be such a topic.

The authors thank Sandra A. Hagen, BS, for her help in extracting the data for this study.

\section{References}

1. National Heart, Lung, and Blood Institute. Seventh Report of the Joint National Committee on Prevention, Detection, Evaluation, and Treatment of High Blood Pressure. Available at http://www.nhlbi.nih. gov/guidelines/hypertension/jnc7full.htm. Accessed November 17, 2011.

2. Messerli FH, Bangalore S. Half a century of hydrochlorothiazide: facts, fads, fiction, and follies. Am J Med 2011;124:896-9.

3. Dorsch MP, Gillespie BW, Erickson SR, Bleske BE, Weder AB. Chlorthalidone reduces cardiovascular events compared with hydrochlorothiazide: a retrospective cohort analysis. Hypertension 2011;57:689-94.

4. Ernst ME, Carter BL. Renewed interest in chlorthalidone: evidence from the Veterans Health Administration. J Clin Hyperten 2010;12:927-34.

5. Ernst ME, Carter BL, Zheng S, Grimm RH, Metaanalysis of dose-response characteristics of hydrochlorothiazide and chlorthalidone: effects on systolic blood pressure and potassium. Am J Hypertens 2010; 23:440-6.

6. Al Badarin FJ, Abuannadi MA, Lavie CJ, O'Keefe JH. Evidence-based diuretic therapy for improving cardiovascular prognosis in systemic hypertension. Am J Cardiol 2011;107:1178-84.

7. Taler SJ. Should chlorthalidone be the diuretic of choice for antihypertensive therapy? Curr Hypertens Rep 2008;10:293-7.

8. Hagen MD, Ivins D, Puffer JC, et al. Maintenance of certification for family physicians (MC-FP) self-assessment modules (SAMs): the first year. J Am Board Fam Med 2006;19:398-403.

9. Haines A, Jones R. Implementing findings of research. BMJ 1994;308:1488-92.

10. Ernst ME, Neaton JD, Grimm RH Jr, et al; Multiple Risk Factor Intervention Trial Research Group. Long-term effects of chlorthalidone versus hydrochlorothiazide on electrocardiographic left ventricular hypertrophy in the multiple risk factor intervention trial. Hypertension 2011;58:1001-7.

11. Prevention of stroke by antihypertensive drug treatment in older persons with isolated systolic hypertension. Final results of the Systolic Hypertension in the Elderly Program (SHEP). SHEP Cooperative Research Group. JAMA 1991;265:3255-64.

12. The treatment of mild hypertension study. A randomized, placebo-controlled trial of a nutritional-hygienic regimen along with various drug monotherapies. The Treatment of Mild Hypertension Research Group. Arch Intern Med 1991;151:1413-23. 
13. ALLHAT Officers and Coordinators for the ALLHAT Collaborative Research Group. The Antihypertensive and Lipid-Lowering Treatment to Prevent Heart Attack Trial. Major outcomes in high-risk hypertensive patients randomized to angiotensin-converting enzyme inhibitor or calcium channel blocker vs diuretic: The Antihypertensive and Lipid-Lowering Treatment to Prevent Heart Attack Trial (ALLHAT). JAMA 2002; 288:2981-97.

14. Ernst ME, Carter BL, Goerdt CJ, et al. Comparative antihypertensive effects of hydrochlorothiazide and chlorthalidone on ambulatory and office blood pressure. Hypertension 2006;47:352-8.

15. Kostis JB, Cabrera J, Cheng JQ, et al. Association between chlorthalidone treatment of systolic hypertension and long-term survival. JAMA 2011;306:2588-93.
16. Carter BL, Ernst ME, Cohen JD. Hydrochlorothiazide versus chlorthalidone: evidence supporting their interchangeability. Hypertension 2004;43:4-9.

17. Wiysonge CS, Bradley HA, Volmink J, Mayosi BM, Mbewu A, Opie LH. Beta-blockers for hypertension. Cochrane Database Syst Rev 2007;8:CD002003.

18. Franse LV, Pahor M, Di Bari M, Somes GW, Cuhsman WC, Applegate WB. Hypokalemia associated with diuretic use and cardiovascular events in the Systolic Hypertension in the Elderly Program. Hypertension 2000;35:1025-30.

19. Sumner W 2nd, O'Neill TR, Roussel G, et al. Family physicians' completion of scoring criteria in virtual patient encounters. AMIA Annu Symp Proc 2011;2011:1355-60. 